\title{
A Novel Anticancer Organic Drug Based on the Natural Product Synthesis
}

\section{T Hannah Clara $^{1,2}$ and Johanan Christian Prasana ${ }^{1 *}$}

${ }^{1}$ Department of Physics, Madras Christian College, Chennai, India

${ }^{2}$ University of Madras, Chennai, India

*Corresponding Author: Johanan Christian Prasana, Department of Physics, Madras Christian College, Chennai, India.
Received: August 26, 2021

Published: September 18, 2021

(C) All rights are reserved by T Hannah Clara and Johanan Christian Prasana.

\begin{abstract}
Despite the outstanding ongoing research on anticancer activity the limited availability from the natural resources has barred drug discovery in researchers. The present work has been carried in achieving 99 percent pure synthesized chalcone derivative (2E)1-(4-aminophenyl)-3-(4-benzyloxyphenyl)-prop-2-en-1-one (APBPP) with simple, cost effective method under room temperature conditions. As per the unique results obtained APBPP significantly augment anticancer effects theoretically.
\end{abstract}

Keywords: DFT; Chalcones; Anticancer; Docking

\section{Introduction}

The past few decades have seen a rapid rise in cancer research, particularly in chemicals which exhibit proactive and degenerative biological behavior when interacting with cancerous tissues. Chalcones have proven to be a very promising class of chemicals in such research. Chalcones are polyphenolic, plant-derived compounds belonging to the flavonoid family, they are aromatic ketones and enones and have shown promising antineoplastic properties on inspections through infrared spectroscopy and DFT calculations [1]. The chalconoid Butein which can be found in Toxicodendron verinicifluum, Dahlia, Butea and Coreopsis has displayed antioxidative and anti-cancer properties, it has also displayed a high ability to inhibit and/or control aromatase process in the human body and hence its effects of breast cancer and estrogen production are being thoroughly studied [2]. In addition, the apigenin falvone glucoside Vitexin found in the passion flower, Vitex agnus castus (Chaste tree) and bamboo (Phyllostachys nigra) has displayed anti-tumor and degenerative properties when dealing with cancerous cells and the chemical 2,4 dihydroxychalcone [3] isolated from Herba oxytropis has displayed a high pro-apoptosis properties induc- ing large scale cell death when interacting with cancer cells and has been studied widely as a potential treatment for prostate cancer. In this study we aim to compile research data regarding the biological and pharmacological activities of the chalcone (2E)-1-(4aminophenyl)-3-(4-benzyloxyphenyl)-prop-2-en-1-one (APBPP) and to reach a scientifically viable statistical conclusion about their general effectiveness in cancer treatment and therapy.

Experimental procedure

The title compound was synthesised by Claisenschmidt condensation reaction method. And the reported procedure is as follows : In a $250 \mathrm{ml}$ beaker the sample was stirred well for re-crystallization with cost effective solvent ethanol and was kept for drying [4].

\section{Computational details}

The structure of the compound was optimised using B3LYP/6$31++G(d, p)$ level of theory with Gaussian 09 software [5] and Mercury crystal structure visualisation (3.8) software. The compound was virtually analysed using Auto dock tools [6] to investigate the pharmaceutical nature of the title compound. 


\section{Results and Discussion}

Computational geometry

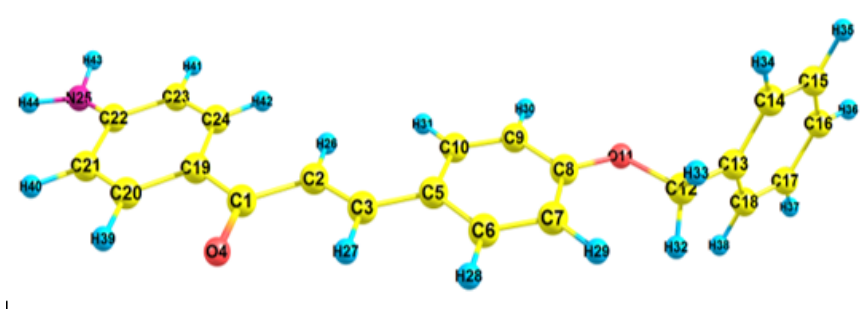

Figure 1: Optimized geometric structure of APBPP.

The B3LYP optimised structure using B3LYP/6-31++G(d,p) basis set is presented in Fig 1 . The $\mathrm{x}, \mathrm{y}, \mathrm{z}$ planes shows identity and the $1 / 2-x, 1 / 2+y, 1 / 2-z$ planes shows screw fold (2-axis) symmetry with direction $[0,1,0]$ at $1 / 4, y, 1 / 4$ with screw component $[0,1 / 2,0]$. The compound exhibits inversion at $[0,0,0]$ in $-x,-y,-z$ directions. A maximum torsion angle of $179^{\circ} \mathrm{A}$ is observed at $\mathrm{C} 1, \mathrm{C} 5, \mathrm{C} 7, \mathrm{C} 12, \mathrm{C} 13, \mathrm{C} 17, \mathrm{C} 20, \mathrm{H} 6, \mathrm{H} 11$ and H12atoms and is shown in table 1 . The experimental and theoretical bond length and bond angles are in good agreement with each other [7].

\begin{tabular}{|c|c|c|c|c|c|}
\hline Number & Atom1 & Atom2 & Atom3 & Atom4 & Torsion \\
\hline 1 & C6 & C1 & C2 & H2 & -179.7 \\
\hline 2 & C6 & C1 & C2 & C3 & $0.3(5)$ \\
\hline 3 & N1 & C1 & C2 & H2 & 1 \\
\hline 4 & N1 & C1 & C2 & C3 & $-179.0(3)$ \\
\hline 5 & C2 & C1 & C6 & C5 & $0.2(5)$ \\
\hline 6 & C2 & C1 & C6 & H6 & -179.8 \\
\hline 7 & N1 & C1 & C6 & C5 & $179.5(4)$ \\
\hline 8 & N1 & C1 & C6 & H6 & -0.5 \\
\hline 9 & C2 & C1 & N1 & H1B & $-12(3)$ \\
\hline 10 & C2 & C1 & N1 & H1A & $-173(4)$ \\
\hline 11 & C6 & C1 & N1 & H1B & $168(3)$ \\
\hline 12 & C6 & C1 & N1 & H1A & $8(4)$ \\
\hline 13 & C1 & C2 & C3 & H3 & -179.9 \\
\hline 14 & C1 & C2 & C3 & C4 & $0.2(5)$ \\
\hline 15 & H2 & C2 & C3 & H3 & 0.1 \\
\hline 16 & H2 & C2 & C3 & C4 & -179.9 \\
\hline 17 & C2 & C3 & C4 & C5 & $-1.1(4)$ \\
\hline 18 & C2 & C3 & C4 & C7 & $179.1(3)$ \\
\hline 19 & H3 & C3 & C4 & C5 & 179 \\
\hline 20 & H3 & C3 & C4 & C7 & -0.9 \\
\hline 21 & C3 & C4 & C5 & H5 & -178.4 \\
\hline & & & & & \\
\hline
\end{tabular}

\begin{tabular}{|c|c|c|c|c|c|}
\hline 22 & $\mathrm{C} 3$ & $\mathrm{C} 4$ & $\mathrm{C} 5$ & $\mathrm{C} 6$ & $1.5(4)$ \\
\hline 23 & $\mathrm{C} 7$ & $\mathrm{C} 4$ & $\mathrm{C} 5$ & H5 & 1.4 \\
\hline 24 & $\mathrm{C} 7$ & $\mathrm{C} 4$ & $\mathrm{C} 5$ & C6 & $-178.6(3)$ \\
\hline 25 & $\mathrm{C} 3$ & $\mathrm{C} 4$ & $\mathrm{C} 7$ & $\mathrm{C} 8$ & $-172.4(3)$ \\
\hline 26 & $\mathrm{C} 3$ & $\mathrm{C} 4$ & $\mathrm{C} 7$ & 01 & $5.6(4)$ \\
\hline 27 & $\mathrm{C} 5$ & $\mathrm{C} 4$ & $\mathrm{C} 7$ & $\mathrm{C} 8$ & $7.8(4)$ \\
\hline 28 & $\mathrm{C} 5$ & $\mathrm{C} 4$ & $\mathrm{C} 7$ & 01 & $-174.2(3)$ \\
\hline 29 & $\mathrm{C} 4$ & $\mathrm{C} 5$ & $\mathrm{C} 6$ & $\mathrm{C} 1$ & $-1.2(5)$ \\
\hline 30 & $\mathrm{C} 4$ & $\mathrm{C} 5$ & $\mathrm{C} 6$ & H6 & 178.8 \\
\hline 31 & H5 & $\mathrm{C} 5$ & $\mathrm{C} 6$ & $\mathrm{C} 1$ & 178.8 \\
\hline 32 & H5 & $\mathrm{C} 5$ & $\mathrm{C} 6$ & H6 & -1.2 \\
\hline 33 & $\mathrm{C} 4$ & $\mathrm{C} 7$ & $\mathrm{C} 8$ & H8 & -17.3 \\
\hline 34 & $\mathrm{C} 4$ & $\mathrm{C} 7$ & $\mathrm{C} 8$ & $\mathrm{C} 9$ & $162.7(3)$ \\
\hline 35 & 01 & $\mathrm{C} 7$ & $\mathrm{C} 8$ & H8 & 164.7 \\
\hline 36 & 01 & $\mathrm{C} 7$ & C8 & $\mathrm{C} 9$ & $-15.4(5)$ \\
\hline 37 & $\mathrm{C} 7$ & $\mathrm{C} 8$ & $\mathrm{C} 9$ & H9 & 2.4 \\
\hline 38 & $\mathrm{C} 7$ & $\mathrm{C} 8$ & $\mathrm{C} 9$ & $\mathrm{C} 10$ & $-177.7(3)$ \\
\hline 39 & H8 & $\mathrm{C} 8$ & C9 & H9 & -177.7 \\
\hline 40 & H8 & $\mathrm{C} 8$ & C9 & $\mathrm{C} 10$ & 2.2 \\
\hline 41 & C8 & C9 & C10 & C11 & $-3.7(5)$ \\
\hline 42 & $\mathrm{C} 8$ & $\mathrm{C} 9$ & $\mathrm{C} 10$ & C15 & $175.3(3)$ \\
\hline 43 & H9 & $\mathrm{C} 9$ & $\mathrm{C} 10$ & C11 & 176.2 \\
\hline 44 & H9 & $\mathrm{C} 9$ & $\mathrm{C} 10$ & C15 & -4.8 \\
\hline 45 & $\mathrm{C} 9$ & $\mathrm{C} 10$ & $\mathrm{C} 11$ & H11 & -2.1 \\
\hline 46 & C9 & C10 & C11 & C12 & $177.8(3)$ \\
\hline 47 & $\mathrm{C} 15$ & $\mathrm{C} 10$ & C11 & H11 & 178.9 \\
\hline 48 & $\mathrm{C} 15$ & $\mathrm{C} 10$ & C11 & C12 & $-1.2(5)$ \\
\hline 49 & $\mathrm{C} 9$ & $\mathrm{C} 10$ & $\mathrm{C} 15$ & $\mathrm{C} 14$ & $-178.2(3)$ \\
\hline 50 & $\mathrm{C} 9$ & $\mathrm{C} 10$ & $\mathrm{C} 15$ & H15 & 1.8 \\
\hline 51 & C11 & $\mathrm{C} 10$ & C15 & C14 & $0.9(5)$ \\
\hline 52 & C11 & $\mathrm{C} 10$ & $\mathrm{C} 15$ & H15 & -179.2 \\
\hline 53 & $\mathrm{C} 10$ & $\mathrm{C} 11$ & C12 & H12 & 179.9 \\
\hline 54 & $\mathrm{C} 10$ & $\mathrm{C} 11$ & $\mathrm{C} 12$ & $\mathrm{C} 13$ & $-0.1(6)$ \\
\hline 55 & H11 & $\mathrm{C} 11$ & $\mathrm{C} 12$ & H12 & -0.1 \\
\hline 56 & H11 & C11 & $\mathrm{C} 12$ & $\mathrm{C} 13$ & 179.8 \\
\hline 57 & C11 & $\mathrm{C} 12$ & $\mathrm{C} 13$ & C14 & $1.7(5)$ \\
\hline 58 & C11 & C12 & C13 & 02 & $-172.0(4)$ \\
\hline 59 & H12 & $\mathrm{C} 12$ & $\mathrm{C} 13$ & C14 & -178.3 \\
\hline 60 & H12 & $\mathrm{C} 12$ & $\mathrm{C} 13$ & 02 & 8 \\
\hline 61 & $\mathrm{C} 12$ & $\mathrm{C} 13$ & C14 & H14 & 178.1 \\
\hline 62 & $\mathrm{C} 12$ & $\mathrm{C} 13$ & $\mathrm{C} 14$ & C15 & $-2.0(5)$ \\
\hline 63 & 02 & C13 & $\mathrm{C} 14$ & H14 & -9.7 \\
\hline 64 & 02 & $\mathrm{C} 13$ & C14 & C15 & $170.3(4)$ \\
\hline 65 & $\mathrm{C} 12$ & $\mathrm{C} 13$ & 02 & C16 & $-179.3(4)$ \\
\hline 66 & $\mathrm{C} 14$ & $\mathrm{C} 13$ & 02 & C16 & $7.8(7)$ \\
\hline 67 & C13 & C14 & C15 & C10 & $0.7(5)$ \\
\hline 68 & $\mathrm{C} 13$ & $\mathrm{C} 14$ & $\mathrm{C} 15$ & H15 & -179.3 \\
\hline 69 & H14 & $\mathrm{C} 14$ & C15 & $\mathrm{C} 10$ & -179.3 \\
\hline 70 & H14 & $\mathrm{C} 14$ & C15 & H15 & 0.7 \\
\hline 71 & C13 & 02 & C16 & H16A & 58.5 \\
\hline
\end{tabular}




\begin{tabular}{|c|c|c|c|c|c|}
\hline 72 & C13 & O2 & C16 & H16B & -60.2 \\
\hline 73 & C13 & O2 & C16 & C17 & $179.1(5)$ \\
\hline 74 & O2 & C16 & C17 & C18 & $-38.2(8)$ \\
\hline 75 & O2 & C16 & C17 & C22 & $142.2(8)$ \\
\hline 76 & H16A & C16 & C17 & C18 & 82.2 \\
\hline 77 & H16A & C16 & C17 & C22 & -97.3 \\
\hline 78 & H16B & C16 & C17 & C18 & -159 \\
\hline 79 & H16B & C16 & C17 & C22 & 21 \\
\hline 80 & C16 & C17 & C18 & H18 & 4 \\
\hline 81 & C16 & C17 & C18 & C19 & $-176.4(7)$ \\
\hline 82 & C22 & C17 & C18 & H18 & -176.7 \\
\hline 83 & C22 & C17 & C18 & C19 & $3(1)$ \\
\hline 84 & C16 & C17 & C22 & C21 & $176.4(8)$ \\
\hline 85 & C16 & C17 & C22 & H22 & -4 \\
\hline 86 & C18 & C17 & C22 & C21 & $-3(1)$ \\
\hline 87 & C18 & C17 & C22 & H22 & 176.8 \\
\hline 88 & C17 & C18 & C19 & H19 & 179.6 \\
\hline 89 & C17 & C18 & C19 & C20 & $-0(1)$ \\
\hline 90 & H18 & C18 & C19 & H19 & 0 \\
\hline
\end{tabular}

\begin{tabular}{|c|c|c|c|c|c|}
\hline 119 & $\mathrm{H} 24$ & C24 & $\mathrm{C} 25$ & $\mathrm{C} 26$ & -179.8 \\
\hline 120 & C24 & $\mathrm{C} 25$ & $\mathrm{C} 26$ & C27 & $-0.9(4)$ \\
\hline 121 & C24 & C25 & C26 & C29 & $179.7(2)$ \\
\hline 122 & H25 & C25 & C26 & C27 & 179 \\
\hline 123 & $\mathrm{H} 25$ & $\mathrm{C} 25$ & $\mathrm{C} 26$ & C29 & -0.4 \\
\hline 124 & C25 & C26 & C27 & $\mathrm{H} 27$ & -178.5 \\
\hline 125 & $\mathrm{C} 25$ & C26 & C27 & C28 & $1.5(4)$ \\
\hline 126 & C29 & C26 & C27 & $\mathrm{H} 27$ & 0.9 \\
\hline 127 & C29 & C26 & $\mathrm{C} 27$ & C28 & $-179.1(2)$ \\
\hline 128 & C25 & C26 & C29 & C30 & $164.7(2)$ \\
\hline 129 & C25 & C26 & C29 & 03 & $-12.8(4)$ \\
\hline 130 & C27 & C26 & C29 & C30 & $-14.6(4)$ \\
\hline 131 & C27 & C26 & C29 & 03 & $167.8(3)$ \\
\hline 132 & C26 & C27 & C28 & $\mathrm{C} 23$ & $-1.2(4)$ \\
\hline 133 & C26 & C27 & C28 & $\mathrm{H} 28$ & 178.8 \\
\hline 134 & $\mathrm{H} 27$ & C27 & C28 & C23 & 178.8 \\
\hline 135 & $\mathrm{H} 27$ & C27 & C28 & $\mathrm{H} 28$ & -1.2 \\
\hline 136 & C26 & C29 & C30 & H30 & 14.7 \\
\hline 137 & C26 & C29 & C30 & C31 & $-165.3(2)$ \\
\hline 138 & 03 & C29 & C30 & H30 & -167.8 \\
\hline 139 & 03 & C29 & C30 & C31 & $12.2(4)$ \\
\hline 140 & C29 & C30 & C31 & H31 & -1.3 \\
\hline 141 & C29 & C30 & C31 & C32 & $178.7(2)$ \\
\hline 142 & $\mathrm{H} 30$ & C30 & C31 & $\mathrm{H} 31$ & 178.7 \\
\hline 143 & $\mathrm{H} 30$ & C30 & C31 & C32 & -1.3 \\
\hline 144 & C30 & C31 & C32 & C33 & $-165.2(3)$ \\
\hline 145 & C30 & C31 & C32 & C37 & $15.2(4)$ \\
\hline 146 & H31 & C31 & C32 & C33 & 14.8 \\
\hline 147 & H31 & C31 & C32 & C37 & -164.8 \\
\hline 148 & C31 & C32 & C33 & H33 & 0.4 \\
\hline 149 & C31 & C32 & C33 & C34 & $-179.6(2)$ \\
\hline 150 & C37 & C32 & C33 & H33 & -179.9 \\
\hline 151 & C37 & C32 & C33 & C34 & $0.0(4)$ \\
\hline 152 & C31 & C32 & C37 & C36 & $178.6(2)$ \\
\hline 153 & C31 & C32 & C37 & H37 & -1.4 \\
\hline 154 & C33 & C32 & C37 & C36 & $-1.1(4)$ \\
\hline 155 & C33 & C32 & C37 & H37 & 179 \\
\hline 156 & C32 & C33 & C34 & H34 & -178.9 \\
\hline 157 & C32 & C33 & C34 & C35 & 1.1(4) \\
\hline 158 & $\mathrm{H} 33$ & C33 & C34 & H34 & 1.1 \\
\hline 159 & H33 & C33 & C34 & C35 & -179 \\
\hline 160 & C33 & C34 & C35 & C36 & $-1.1(4)$ \\
\hline 161 & C33 & C34 & C35 & 04 & $177.5(2)$ \\
\hline 162 & $\mathrm{H} 34$ & C34 & C35 & C36 & 178.8 \\
\hline 163 & H34 & C34 & C35 & 04 & -2.5 \\
\hline 164 & C34 & C35 & C36 & H36 & -180 \\
\hline 165 & C34 & C35 & C36 & C37 & $0.1(4)$ \\
\hline 166 & 04 & C35 & C36 & H36 & 1.3 \\
\hline 167 & 04 & C35 & C36 & C37 & $-178.7(2)$ \\
\hline
\end{tabular}

\begin{tabular}{|c|c|c|c|c|c|}
\hline Number & Atom1 & Atom2 & Atom3 & Atom4 & Torsion \\
\hline 91 & H18 & C18 & C19 & C20 & 179.6 \\
\hline 92 & C18 & C19 & C20 & H20 & 177 \\
\hline 93 & C18 & C19 & C20 & C21 & $-3(2)$ \\
\hline 94 & H19 & C19 & C20 & H20 & -3 \\
\hline 95 & H19 & C19 & C20 & C21 & 177 \\
\hline 96 & C19 & C20 & C21 & H21 & -177 \\
\hline 97 & C19 & C20 & C21 & C22 & $3(2)$ \\
\hline 98 & H20 & C20 & C21 & H21 & 3 \\
\hline 99 & H20 & C20 & C21 & C22 & -177 \\
\hline 100 & C20 & C21 & C22 & C17 & $0(1)$ \\
\hline 101 & C20 & C21 & C22 & H22 & -180 \\
\hline 102 & H21 & C21 & C22 & C17 & -179.7 \\
\hline 103 & H21 & C21 & C22 & H22 & 0 \\
\hline 104 & C28 & C23 & C24 & H24 & -179.9 \\
\hline 105 & C28 & C23 & C24 & C25 & $0.2(4)$ \\
\hline 106 & N2 & C23 & C24 & H24 & -1.9 \\
\hline 107 & N2 & C23 & C24 & C25 & $178.2(3)$ \\
\hline 108 & C24 & C23 & C28 & C27 & $0.4(4)$ \\
\hline 109 & C24 & C23 & C28 & H28 & -179.7 \\
\hline 110 & N2 & C23 & C28 & C27 & $-177.7(3)$ \\
\hline 111 & N2 & C23 & C28 & H28 & 2.3 \\
\hline 112 & C24 & C23 & N2 & H2B & $170(3)$ \\
\hline 113 & C24 & C23 & N2 & H2A & $13(3)$ \\
\hline 114 & C28 & C23 & N2 & H2B & $-12(3)$ \\
\hline 115 & C28 & C23 & N2 & H2A & $-169(3)$ \\
\hline 116 & C23 & C24 & C25 & H25 & -179.8 \\
\hline 117 & C23 & C24 & C25 & C26 & $0.1(4)$ \\
\hline 118 & H24 & C24 & C25 & H25 & 0.2 \\
\hline
\end{tabular}




\begin{tabular}{|c|c|c|c|c|c|}
\hline Number & Atom1 & Atom2 & Atom3 & Atom4 & Torsion \\
\hline 168 & C34 & C35 & 04 & C38 & $5.8(4)$ \\
\hline 169 & C36 & C35 & 04 & C38 & $-175.5(2)$ \\
\hline 170 & C35 & C36 & C37 & C32 & $1.0(4)$ \\
\hline 171 & C35 & C36 & C37 & H37 & -179 \\
\hline 172 & H36 & C36 & C37 & C32 & -178.9 \\
\hline 173 & H36 & $\mathrm{C} 36$ & C37 & H37 & 1 \\
\hline 174 & H38A & C38 & C39 & $\mathrm{C} 40$ & -65.7 \\
\hline 175 & H38A & C38 & C39 & $\mathrm{C} 44$ & 115.8 \\
\hline 176 & H38B & C38 & C39 & $\mathrm{C} 40$ & 52.8 \\
\hline 177 & H38B & C38 & C39 & C44 & -125.7 \\
\hline 178 & 04 & C38 & C39 & $\mathrm{C} 40$ & $173.5(3)$ \\
\hline 179 & 04 & C38 & C39 & $\mathrm{C} 44$ & $-5.0(4)$ \\
\hline 180 & H38A & C38 & 04 & $\mathrm{C} 35$ & 66.3 \\
\hline 181 & H38B & C38 & 04 & C35 & -52.3 \\
\hline 182 & C39 & C38 & 04 & C35 & $-173.0(2)$ \\
\hline 183 & C38 & C39 & $\mathrm{C} 40$ & $\mathrm{H} 40$ & 1.5 \\
\hline 184 & C38 & C39 & $\mathrm{C} 40$ & $\mathrm{C} 41$ & $-178.5(3)$ \\
\hline 185 & $\mathrm{C} 44$ & C39 & $\mathrm{C} 40$ & $\mathrm{H} 40$ & -179.9 \\
\hline 186 & $\mathrm{C} 44$ & C39 & $\mathrm{C} 40$ & $\mathrm{C} 41$ & $0.1(5)$ \\
\hline 187 & C38 & C39 & $\mathrm{C} 44$ & $\mathrm{C} 43$ & 178.3(3) \\
\hline 188 & C38 & C39 & C44 & $\mathrm{H} 44$ & -1.7 \\
\hline 189 & $\mathrm{C} 40$ & C39 & C44 & $\mathrm{C} 43$ & $-0.2(5)$ \\
\hline 190 & $\mathrm{C} 40$ & C39 & $\mathrm{C} 44$ & $\mathrm{H} 44$ & 179.8 \\
\hline 191 & C39 & $\mathrm{C} 40$ & $\mathrm{C} 41$ & H41 & 179.4 \\
\hline 192 & C39 & $\mathrm{C} 40$ & $\mathrm{C} 41$ & $\mathrm{C} 42$ & $-0.7(5)$ \\
\hline 193 & $\mathrm{H} 40$ & $\mathrm{C} 40$ & $\mathrm{C} 41$ & H41 & -0.7 \\
\hline 194 & $\mathrm{H} 40$ & $\mathrm{C} 40$ & C41 & $\mathrm{C} 42$ & 179.3 \\
\hline 195 & $\mathrm{C} 40$ & $\mathrm{C} 41$ & $\mathrm{C} 42$ & $\mathrm{H} 42$ & -178.8 \\
\hline 196 & $\mathrm{C} 40$ & $\mathrm{C} 41$ & $\mathrm{C} 42$ & $\mathrm{C} 43$ & $1.3(6)$ \\
\hline 197 & H41 & $\mathrm{C} 41$ & $\mathrm{C} 42$ & $\mathrm{H} 42$ & 1.2 \\
\hline 198 & H41 & $\mathrm{C} 41$ & $\mathrm{C} 42$ & $\mathrm{C} 43$ & -178.8 \\
\hline 199 & $\mathrm{C} 41$ & $\mathrm{C} 42$ & $\mathrm{C} 43$ & $\mathrm{H} 43$ & 178.6 \\
\hline 200 & $\mathrm{C} 41$ & $\mathrm{C} 42$ & $\mathrm{C} 43$ & $\mathrm{C} 44$ & $-1.3(5)$ \\
\hline 201 & $\mathrm{H} 42$ & $\mathrm{C} 42$ & $\mathrm{C} 43$ & $\mathrm{H} 43$ & -1.4 \\
\hline 202 & $\mathrm{H} 42$ & $\mathrm{C} 42$ & $\mathrm{C} 43$ & $\mathrm{C} 44$ & 178.7 \\
\hline 203 & $\mathrm{C} 42$ & $\mathrm{C} 43$ & $\mathrm{C} 44$ & C39 & $0.8(5)$ \\
\hline 204 & $\mathrm{C} 42$ & $\mathrm{C} 43$ & $\mathrm{C} 44$ & $\mathrm{H} 44$ & -179.2 \\
\hline 205 & $\mathrm{H} 43$ & $\mathrm{C} 43$ & $\mathrm{C} 44$ & C39 & -179.1 \\
\hline 206 & $\mathrm{H} 43$ & $\mathrm{C} 43$ & $\mathrm{C} 44$ & H44 & 0.9 \\
\hline
\end{tabular}

Table 1: Torsion angles of APBPP.

Molecular docking

Molecular docking was done to predict the best binding conformation of ligand to a macromolecule. Here in the best pose ligands for four different cancer were selected from their lowest binding energy and presented [8]. The crystal structure of 5JMS (Liver cancer) [9], 5W2L (Bone cancer) [10], 6DYV (stomach cancer) [11], 6LU4(Breast cancer) [12] were derived from RCSB Protien data bank. All the water, co-crystallized inhibitors and interacting ions were removed and polar hydrogens were added also the charges namely Kollman and Gasteiger were obtained to examine the PDBQT files. Using Grid box centres were confirmed in Autodock tools. The title chalcone acts as ligand and the docking were done to various types of cancer proteins of which the best conformations are only presented in this study. The APBPP molecule in comparison with the approved drug Erlotinib is tabulated. From the Table 2 it was observed that the binding energy of APBPP is comparable with Erlotinib and hence could be of use clinically. The structure of the standard drug was obtained from Drug bank database. The ligand with torsion information was added and saved as PDBQT file. The synthesized chalcone binds tightly into the inhibition sites of all types of cancer and is comparable with that of the approved drug Erlotinib. These results portray the pharmocopore nature of the title compound to be a suitable drug for various types of Liver, Bone, stomach and Breast cancer treatment. This work can be further extended with experimental analysis to validate theoretical results.

\begin{tabular}{|c|c|c|c|c|}
\hline $\begin{array}{l}\text { Protein } \\
\text { PDB }\end{array}$ & $\begin{array}{l}\text { Bonded } \\
\text { residue }\end{array}$ & $\begin{array}{c}\text { Hydrogen } \\
\text { bonds }\end{array}$ & $\begin{array}{c}\text { Binding } \\
\text { Energy } \\
\text { (Kcal/mol) }\end{array}$ & $\begin{array}{c}\text { Inhibition- } \\
\text { constant } \\
\text { ( } \mu \text { mol) }\end{array}$ \\
\hline 5JMS & $\begin{array}{l}\text { ARG30, } \\
\text { LIG1:H }\end{array}$ & 2 & -5.83 & 151.76 \\
\hline $5 W 2 L$ & $\begin{array}{l}\text { PHE80, } \\
\text { GLU80 }\end{array}$ & 2 & -3.57 & $2.42 \mathrm{mMol}$ \\
\hline 6DYV & $\begin{array}{l}\text { CY52:H, } \\
\text { GLY3:H }\end{array}$ & 2 & -4.22 & 800.04 \\
\hline 6LU4 & $\begin{array}{l}\text { GLY227, } \\
\text { LIG1:H, } \\
\text { LEU225 }\end{array}$ & 3 & -4.93 & 242.78 \\
\hline $\begin{array}{c}\text { Erlotinib } \\
\text { (Drug) }\end{array}$ & $\begin{array}{c}\text { LEU654, } \\
\text { GLY678 } \\
\text { GLY684, } \\
\text { THR759 }\end{array}$ & 4 & -7.11 & $2.67 \mathrm{mMol}$ \\
\hline
\end{tabular}

Table 2: Docking parameters of APBPP. 

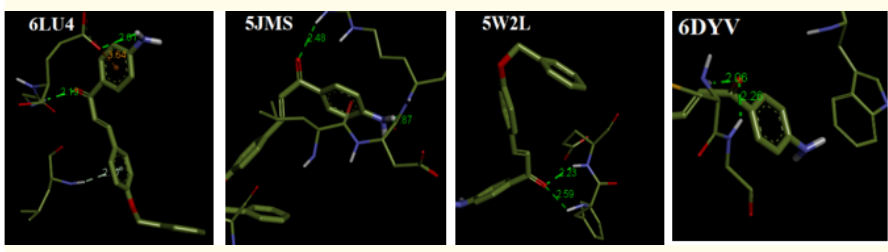

Figure 2: Docking conformations of APBPP.

\section{Conclusion}

Cancer active pharmocopore was synthesized using simple Claisen-Schmidt method. The synthesis of the title compound is easily scalable for large scale manufacture, biocompatible and economically feasible. The present study explores the wide potent anticancer activity of the title compound with molecular docking studies and compared with standard drug using Auto dock tools. The results observed showcases the title chalcone as an effective anticancer drug in pharmaceutical industry with minimal side effects as the product is of organic nature. Hence this work paves way for the researchers to confine their drug design according to their need in anticancer therapy by modifying the positioning of functional groups in the title compound.

\section{Bibliography}

1. Mohapatra Ranjan K., et al. "DFT, anticancer, antioxidant and molecular docking investigations of some ternary Ni (II) complexes with 2-(E)-4-(dimethylamino) phenyl. methyleneamino. phenol". Chemical Papers 75.3 (2021): 1005-1019.

2. Hashemzadeh Hassan and Heidar Raissi. "Covalent organic framework as smart and high efficient carrier for anticancer drug delivery: a DFT calculations and molecular dynamics simulation study". Journal of Physics D: Applied Physics 51.34 (2018): 345401.

3. Lou Chenghua., et al. "Preliminary studies on anti-tumor activity of 2', 4'-dihydroxychalcone isolated from Herba Oxytropis in human gastric cancer MGC-803 cells". Toxicology in Vitro 23.5 (2009): 906-910.

4. Nasir Abbas Bukhari., et al. "Synthesis and biological evaluation of chalcone derivatives (mini review)". Mini Reviews in Medicinal Chemistry 12.13 (2012): 1394-1403.
5. RG Parr Yang. "Functional Theory of atoms and molecules". Oxford University Press, New York (1989).

6. Morris G M., et al. Journal of Computational Chemistry 19 (1998): 1639-1662.

7. Clara T., et al. "Quantum mechanical, spectroscopic and docking studies of (2E)-1-(4-aminophenyl)-3-(4-benzyloxyphenyl)prop-2-en-1-one Chalcone derivative by density functional theory-A prospective respiratory drug". Materials Today: Proceedings (2020).

8. Dlala Najet Aouled., et al. "DFT Calculations and Molecular Docking Studies on a Chromene Derivative". Journal of Chemistry 2021 (2021).

9. Tam Leslie., et al. "Expression levels of the JAK/STAT pathway in the transition from hormone-sensitive to hormone-refractory prostate cancer". British Journal of Cancer 97.3 (2007): 378-383.

10. Nguyen Dinh-Duc., et al. "Roles of OB-fold proteins in replication stress". Frontiers in Cell and Developmental Biology 8 (2020): 954.

11. ESMERAY Furkan and İbrahim Hanifi ÖZERCAN. "Mini-Review: The classification studies done for early diagnosis of the stomach cancer". The Online Journal of Science and Technology 8.4 (2018).

12. Robbins K R., et al. "The ant colony algorithm for feature selection in high-dimension gene expression data for disease classification". Mathematical Medicine and Biology: A Journal of the IMA 24.4 (2007): 413-426.

Volume 5 Issue 10 October 2021

(C) All rights are reserved by T Hannah Clara and Johanan Christian Prasana. 